\title{
To register or not to register
}

Dowitcher Research Laboratories (DRL) was a startup contract research laboratory located in a small east coast town. The only animals used by DRL were rabbits that were obtained from a rabbitry in the same town. A local private practice veterinarian was under contract to check the DRL rabbits on a weekly basis. The veterinarian had recently initiated a quarterly health monitoring program for DRL, and no health concerns were identified. All of DRL's business came from private colleges and small research labs in the same state, none of which had an animal facility. DRL did not have an IACUC, nor did DRL inquire as to whether the schools and labs it served had IACUCs.

Eventually the veterinary medical officer from the USDA heard about DRL and visited the site to get a better understanding of why it was not registered and why there was no IACUC overseeing the animal use procedures. The owner politely explained that registration was not required for DRL, according to the Animal Welfare Act. DRL did not participate in interstate commerce ${ }^{1}$, all of its animals were locally bred, it did not receive any federal funds for its research or testing ${ }^{1}$, its animals were healthy and well cared for, and DRL was in compliance with all state regulations.

Does DRL need to be registered with the USDA? If one of the schools it serviced received a federal grant for its rabbit research, or if one of the private labs it worked with was located out-of-state, would that alter the current situation?

1. Animal Welfare Act regulations. 9 CFR. Chapter I, Subchapter A, Part 1, Section 1.1.

\section{RESPONSE}

\section{Where loopholes linger, intent should prevail}

\author{
Sean Maguire, VMD, MS, DACLAM, MRCVS \& \\ Mila C. Kundu, MS, DVM
}

The regulations of the Animal Welfare Act (AWA) require that research facilities be registered with the USDA, so the question at hand is whether Dowitcher Research Laboratories (DRL) is a research facility, as defined by the AWA (\$2132; ref. 1). We know that DRL uses "live animals in research," but does not, to the best of their knowledge, "receive funds... from a department, agency, or instrumentality of the United States," so we must consider whether DRL "purchases or transports live animals in commerce."

The first paragraph of the AWA definition of commerce describes "trade, traffic, transportation, or other commerce" between states. From the perspective of its owner, DRL does not engage in commerce by this definition because all of their research, transactions and parties involved, from their animal suppliers to their customers, are located and remain within the state. The second paragraph of the definition also counts commerce as that "which affects trade, traffic, transportation, or other commerce described in para- graph (1).” Therefore DRL, which is solely engaged in intrastate commerce, might need to be registered with the USDA if its activities affect interstate commerce.

Additionally, the opening of the AWA ${ }^{1}$, states that "animals and activities which are regulated under this chapter are either in interstate or foreign commerce or substantially affect such commerce or the free flow thereof, and that regulation ... is necessary to prevent and eliminate burdens upon such commerce and to effectively regulate such commerce" (\$2131). We take this understanding into consideration, alongside the societal expectation of consistent and appropriate regulation to protect research animals; the history of federal regulations that have been enacted in response to catastrophic or heavily publicized events that resulted in public outrage; and the potentially unfair competitive advantage that DRL could use with less knowledgeable clients. It is our perspective that an unregistered facility such as DRL could substantially affect interstate biomedical research on AWA-regulated species; therefore DRL must register with the USDA.

Pragmatically and regardless of the legal intricacies, the spirit of the AWA demands registration and adherence to the AWA. This entails that DRL have an attending veterinarian and a properly constituted IACUC that approves all institutional research. If we were employed by DRL, we would help them navigate USDA registration and achieve AWA compliance as a mandatory initial professional service. This would significantly broaden their potential client base and ensure their continued ability to meet the needs of current clients, should their circumstances change.

The direct consequences of DRL's unregistered status depend on what enforcement actions the USDA pursues. But if DRL chooses to pursue legal action to defend their position, maintaining that registration is not required because their activity is in-state only, this conflict could draw unfavorable publicity to all involved parties. We are particularly concerned about how DRL and their associated academic and private institutions would handle such unfavorable publicity, which could cause long-term deleterious consequences for the broader research community.

If an institution begins receiving federal grants or clearly engaging in interstate commerce, either by having a client outside of state or transporting animals or materials out of state, that institution is now unambiguously required to register with the USDA and comply with the Animal Welfare Act.

1. Animal Welfare Act as Amended. 7 USC. Chapter 54.

Maguire is a Research Veterinary Manager and Kundu is a Post Doctoral Fellow, Laboratory Animal Sciences at GlaxoSmithKline, Collegeville, PA. 